\title{
THE DOMAIN RANK OF OPEN SURFACES OF INFINITE GENUS
}

\author{
RICHARD J. TONDRA
}

\begin{abstract}
In a recent paper it was shown that an open surface, i.e. a connected 2-manifold without boundary, has finite domain rank if and only if it has finite genus. In the present paper, it is shown that the domain rank of any open surface of infinite genus is countably infinite.
\end{abstract}

1. Introduction. Throughout the presentation, the notation and definitions found in [4] will be used. The principal tool used in this paper is a modification of Theorem 3 of [3]. As in [3], if $M$ is an open surface, let $(X, Y, Z)$ denote the triple of compact totally disconnected spaces determining the ideal boundary of $M$, where $X$ is the entire boundary, $Y$ is the set of nonplanar boundary points, and $Z$ is the set of nonorientable boundary points. In the proof of Theorem 3 of [3], one is mainly concerned with how to identify $C^{+}(k)$ and $C^{-}(k)$ to produce an orientable or nonorientable compact surface. The same result can be obtained by using only $C^{-}(k)$ and properly identifying points of $C^{-}(k)$ to produce a compact surface which is either a handle or möbius band. This alteration has no effect on the validity of the remainder of the proof and the following modification of Theorem 3 of [3] is obtained.

TheOREM 1.1. Let $S^{2}=\left\{x \in R^{3} \mid\|x\|=1\right\}, E^{1}=\left\{x \in S^{2} \mid x_{3} \geqq 0\right\}$, $E^{2}=\left\{x \in S^{2} \mid x_{3} \leqq 0\right\}$, and $S^{1}=E^{1} \cap E^{2}$. Every open surface $M$ is homeomorphic to the surface formed from $S^{2}$ by first removing a closed totally disconnected set $X \subset S^{1}$ from $S^{2}$ and then removing the interiors of a finite or infinite sequence $C_{1}, C_{2}, \ldots$ of pairwise disjoint 2-cells in $E^{2}-S^{1}$ and properly identifying the boundaries of these 2-cells to produce handles or möbius bands. The sequence $C_{1}, C_{2}, \ldots$ "approaches $X^{n}$ in the sense that if $U$ is an open set of $S^{2}, X \subset U$, then all but a finite number of the $C_{i}$ are contained in $U$.

If in forming $M$ via the above theorem, bd $C_{j}$ is identified to produce a handle, $C_{j}$ will be called toral; otherwise, $C_{j}$ will be called projective.

If $M$ is an open surface of infinite genus, then $M$ belongs to one of the following four classes of surfaces: class 1 -orientable surfaces;

Received by the editors January 23, 1970.

AMS 1969 subject classifications. Primary 5701, 5475.

Key words and phrases. Domain, domain rank, surface, ideal boundary. 
class 2-infinitely nonorientable surfaces; class 3-nonorientable surfaces of odd parity; class 4-nonorientable surfaces of even parity. Thus if an open surface $M$ is formed from $S^{2}$ via Theorem 1.1, it may and henceforth will be assumed that if (i) $M \in$ class 1 , each $C_{j}$ is toral; (ii) $M \in$ class $2, C_{1}, C_{2}$, and an infinite number of $C_{j}$ are projective; (iii) $M \in$ class $3, C_{1}$ is projective and all other $C_{j}$ are toral; (iv) $M$ $\in$ class $4, C_{1}, C_{2}$ are projective and all other $C_{j}$ are toral.

2. Proof of the theorem. As in [4], the proof of the theorem depends upon showing that there is an open surface $D$ which is a generator of every open surface $M$ belonging to a certain class of open surfaces. Let $X$ denote the subset of the real line defined by $X$ $=\{0\} \cup\{1 / n \mid n$ is a positive integer $\}$ and let $A \subset X, A=\{0\}$. For each $i, 1 \leqq i \leqq 4$. Let $D_{i}$ denote the open surface of infinite genus belonging to class $i$ such that $(X, A, \varnothing),(X, A, A),(X, A, \varnothing)$, and $(X, A, \varnothing)$ are the ideal boundaries of $D_{1}, D_{2}, D_{3}$, and $D_{4}$ respectively.

THEOREM 2.1. Let $M$ be an open surface of infinite genus, $M \in$ class $q$. Then $D_{q}$ is a generator of $M$.

Proof. Let $M$ be formed from $S^{2}$ via Theorem 1.1 and let $\Gamma$ $=\left\{C_{j}\right\}_{j-1}^{\infty}$ be the sequence of 2-cells which "approaches $X$." Since the sequence "approaches $X$," there exists a subsequence $\Omega=\left\{C_{j}^{\prime}\right\}_{j=1}^{\infty}$ of $\Gamma$ such that $C_{j}^{\prime}=C_{j}, j=1,2 ; C_{j}^{\prime}$ is projective for all $j$ if $q=2$; and $\Omega$ converges to a point $c \in X$. Therefore, there exists a 2-cell $B \subset E^{2}$ such that $B \cap S^{1}=c ; C_{j}^{\prime} C$ int $B, j \geqq 1 ;$ and $C_{j} \cap B \neq \varnothing \Leftrightarrow C_{j} \in \Omega$. Since (1) $W=S^{2}-$ (int $\left.B \cup X\right)$ is homeomorphic to a domain of $H_{2}$ $=\left\{x \in R^{2} \mid x_{2} \geqq 0\right\}$ which contains bd $H_{2}$, (2) $C_{j} \subset$ int $W$ for all $C_{j} \in \Gamma-\Omega$, and (3) $\Gamma$ "approaches $X$," there exists a sequence $\left\{L_{k}\right\}_{k=0}^{\infty}$ of compact surfaces of genus 0 contained in $S^{2}$ such that

(i) $B=L_{0}$;

(ii) $L_{k}=B_{k}-\left(\bigcup_{p=1}^{n(k)}\right.$ int $\left.E_{p}^{k}\right)$ where $B_{k}$ is a 2-cell in $S^{2}$ such that $c \in \mathrm{bd} B_{k}$ and $\left\{E_{p}^{\mathbf{k}}\right\}_{p=1}^{n(k)}$ is a finite disjoint collection of 2-cells contained in int $B_{k}, k \geqq 1$;

(iii) $L_{k} \cap X=c=$ bd $L_{k} \cap X, k \geqq 0$;

(iv) $\left(L_{k}-c\right) \subset$ int $L_{k+1}, k \geqq 0$;

(v) for each $k$, there are at most a finite number of 2-cells $C_{j} \in \Gamma-\Omega$ such that $C_{j} \cap L_{k} \neq \varnothing$, in which case $C_{j} \subset$ int $L_{k}$;

(vi) $S^{2}-X=\cup_{k=1}^{\infty}$ int $L_{k}$.

For each $k \geqq 1$, let $A_{k}$ be a sequence of points in $S^{2}$ which converges to $c$ such that $A_{k} \subset$ int $L_{k}-\left(L_{k-1} \cup\left(\bigcup_{j=1}^{\infty} C_{j}\right)\right)$. Let $Q_{k}=$ int $L_{k}-A_{k}$. Then for all $k \geqq 1, Q_{k}$ is a domain of $S^{2}-X, Q_{k} \subset Q_{k+1}$, and furthermore $S^{2}-X=\bigcup_{k=1}^{\infty} Q_{k}$. Let $p: S^{2}-\left(\bigcup_{j=1}^{\infty}\right.$ int $\left.C_{j}\right) \rightarrow M$ be the identification 
map used in forming $M$ via Theorem 1.1. For each $k \geqq 1$, let $G_{k}$ $=Q_{k}-\left(\bigcup_{j=1}^{\infty}\right.$ int $\left.C_{j}\right)$. Since $C_{j} \cap Q_{k} \neq \varnothing \Leftrightarrow C_{j} \subset Q_{k}$; the set $M_{k}=p\left(G_{k}\right)$ is a domain of $M$. In view of the properties of $\left\{Q_{k}\right\}_{k=1}^{\infty}$, it follows that $M=\mathrm{U}_{k=1}^{\infty} M_{k}$ and $M_{k} \subset M_{k+1}$ for $k \geqq 1$. It follows from (i), (iv), and (v) that for all $k \geqq 1, M_{k} \in$ class $q$. But by (ii), $c \in$ bd $B_{k}$ and the sequence of 2-cells $\Omega$ and the sequence of points $A_{k}$ both converge to $c$. Therefore in view of $(\mathrm{v})$ the ideal boundary $\left(X_{k}, Y_{k}, Z_{k}\right)$ of $M_{k}$ is homeomorphic to the ideal boundary of $D_{q}$. Since $M_{k} \in$ class $q$, it follows from Theorem 1 of [3] that $M_{k} \equiv D_{q}$ for all $k \geqq 1$, and therefore $D_{q}$ is a generator of $M$.

As an immediate consequence we have the following:

THEOREM 2.2. Let $M$ be an open surface. If $M$ has infinite genus, then $D R(M)$ is countably infinite; otherwise, $D R(M)$ is finite.

Proof. Let $M$ be an open surface of infinite genus. If $D$ is a proper domain of $M$, then $D$ is an open surface of infinite or finite genus. If $D$ has infinite genus, then it follows from the previous theorem that $D_{k}$ is a generator of $D$ for some $k, 1 \leqq k \leqq 4$. If $D$ has finite genus, then it follows from Lemma 2.7 of [4] that $Q(\infty)$ is a generator of $D, Q$ a closed surface. Since there are only a countable number of topologically distinct closed surfaces, $D R(M)$ is at most countably infinite. The result now follows from Corollary 2.9 of [4].

Although any open surface has at most countably infinite domain rank, there do exist open 3-manifolds which have noncountable domain rank. Such a 3-manifold $M$ can be constructed from a countable collection $\left\{M_{j}\right\}_{j=1}^{\infty}$ of closed irreducible, orientable 3-manifolds such that $M_{q} \equiv M_{r} \Leftrightarrow q=r$ (see for example [2]), by taking $M$ to be the infinite connected sum of this collection. Then using the results of [1] it can be shown that $D R(M)$ is not countably infinite.

\section{REFERENCES}

1. J. Milnor, A unique decomposition theorem for 3-manifolds, Amer. J. Math. 84 (1962), 1-7. MR 25 \#5518.

2. P. Orlik and F. Raymond, Action of $S O(2)$ on 3-manifolds, Proc. Conference on Transformation Groups, Springer-Verlag, Berlin and New York, 1968.

3. I. Richards, On the classification of noncompact surfaces, Trans. Amer. Math. Soc. 106 (1963), 259-269. MR $26 \# 746$.

4. R. J. Tondra, Characterization of connected 2-manifolds without boundary which have finite domain rank, Proc. Amer. Math. Soc. 22 (1969), 479-482. MR 39 \#6284.

Iowa State University, Ames, Iowa 50010 